\title{
Sick Child Feeding Practice and Associated Factors among Mothers of Children Less Than 24 Months Old, in Burayu Town, Ethiopia
}

\author{
Nega Degefa $\mathbb{D},{ }^{1}$ Hiwot Tadesse, ${ }^{1}$ Fekadu Aga, ${ }^{2}$ and Tomas Yeheyis $\mathbb{D}^{1}$ \\ ${ }^{1}$ Department of Nursing, College of Medicine and Health Science, Arba Minch University, Arba Minch, Ethiopia \\ ${ }^{2}$ Department of Nursing, School of Nursing and Midwifery, College of Medicine and Allied Health Sciences Addis Ababa University, \\ Addis Ababa, Ethiopia
}

Correspondence should be addressed to Nega Degefa; negshd05@gmail.com

Received 17 January 2019; Revised 15 July 2019; Accepted 1 September 2019; Published 22 December 2019

Academic Editor: Samuel Menahem

Copyright (C) 2019 Nega Degefa et al. This is an open access article distributed under the Creative Commons Attribution License, which permits unrestricted use, distribution, and reproduction in any medium, provided the original work is properly cited.

Background. Growing evidence suggests that inadequate intake, poor caring practices, and disease process were some of the immediate and major causes of undernutrition in children. This points out that infant and young child feeding were the basic grounds to improve child survival and promote healthy growth and development. The first two years of a child's life are particularly important, as optimal nutrition during this period lowers morbidity and mortality, reduces the risk of chronic disease, and enhances the chances of better development. The study was aimed to assess sick infant and young child feeding practice and associated factors among mothers of children aged less than 24 months old in the Burayu town Oromia, Ethiopia. Methods. Institutional based cross-sectional study design was utilized. The study was conducted from April-May, 2015 among 362 mother-child pair attending the maternal and childcare $(\mathrm{MCH})$ units of the two public health facilities in the Burayu town. Bivariate and multivariable analysis was done to test the relationship between the explanatory and outcome variables and the odds ratio with $95 \%$ confidence interval and the $p$-value was used to ascertain statistical significance. Result. More than half (53.6\%) of all mothers fed their child more frequently at the time of illness than at a time of health. The mean age of respondents was $25.41 \pm 3.56$ and ranged from $15-30$ years. Nearly three out of five (60.8\%) of the respondents attended no formal education. A mother who had got counseling on sick child feeding were nearly three times more likely to feed their child appropriately than their counterparts (AOR: 2.95; 95\% CI; 1.78, 4.91). Mothers who were housewives were $55 \%$ times less likely to feed their sick child appropriately than those who were working (AOR: 0.45 ; $95 \%$ CI; 0.26, 0.79). Those mothers who have a child aged less than 6 months were $88 \%$ less likely to practice appropriate sick child feeding than those who have a child aged more than 6 months (AOR: 0.22; 95\% CI; 0.12,0.40). Conclusion. Respondents who do not receive counseling on infant and young child feeding have poor sick child feeding practice. Working mother had owned better practices of feeding child particularly at the time of illness. Infants below the age of 6 months deserve more concern in providing frequent breastfeeds at the time of illness.

\section{Background}

Adequate nutrition during infancy and early childhood is the fundamental to the development of each child's full human potential [1]. Optimal infant and young child feeding (IYCF) is found to be essential for child growth. The time during pregnancy and a child's first two years of life is considered a "critical window of opportunity" for the prevention of growth failure [2]. After birth, a child's capacity to achieve the standards in growth will be determined by the sufficiency of dietary intake (which depends on infant and young child feeding and care practices and food security), as well as exposure to diseases [3].

The World health organization (WHO) and the United Nations International Children's Emergency Fund (UNICEF) recommended early initiation of breastfeeding within one hour of birth; exclusive breastfeeding for the first 6 months of life and the introduction of nutritionally adequate and safe complementary foods at 6 months together with continued breastfeeding up to two years of age or beyond $[1,4-6]$. 
Despite the recommendation of the WHO, poor infant feeding practices which are pooled with high rates of infectious diseases all through the first two years of life are the principal contiguous causes of malnutrition. Therefore, it is indispensable to ensure that caregivers are provided with appropriate guidance regarding the optimal feeding of infants and young children [1]. The latest Anthropometric data from low-income countries suggests that the levels of undernutrition increase obviously from 18-24 months to 3 of age [2].

Children's nutritional status can decline rapidly during/ after common childhood illness if the additional nutrient requirements associated with the illness/convalescence are not properly met and the nutrients are averted from growth and development towards building the immune response. Children's poor appetite induced by illness can contribute to preserving the vicious cycle of infection and stunting [7-10].

Existing evidence suggests that, increasing fluid intake during illness, including more frequent breastfeeding and encouraging the child to eat soft, varied, appetizing, and their favorite foods. After the illness, give food more often than usual and encourage the child to eat more [11]. Even though appetite may be reduced, continued feeding of complementary food is acclaimed to preserve nutrient consumption and improve recovery [12]. After the illness, the child needs better nutrient intake to make up for nutrient losses during sickness and allow for catch-up growth. Extra food is required until the child has regained any weight loss and is growing well again.

Furthermore, appropriate infant and young child feeding during and after the illness is part of the Global Strategy for the Integrated Management of Childhood Illnesses and essential nutrition actions promoting maternal newborn, infant and young child nutrition and health $[13,14]$.

Regardless of the well-recognized advantages of breastfeeding worldwide, performance on recommended policies and programs for breastfeeding is poor. No country highly adhere to all of the indicators, proving that significant progress on all fronts is looked-for. Unluckily, countries are not adequately protecting, promoting, or supporting breastfeeding [15].

Despite the overwhelming evidence on the benefits of exclusive breastfeeding, only about one in three African babies under six months are exclusively breastfed, due to the lack of understanding of optimal feeding practices and the lack of support from health care providers, community members, and families. Babies who are not exclusively breastfed in the early months have a higher risk of death, especially from infection [16].

Breastfeeding is nearly universal in Ethiopia and half of the children born in the three years before the survey are breastfed for about 25 months. More than half (52\%) of the children less than 6 months old are exclusively breastfed in Ethiopia [17]. The feeding practices of only $7 \%$ of children in Ethiopia age 6-23 months meet the minimum requirement with respect to all the three IYCF practices (breastfeeding status, number of food groups, and times they were fed during the day or night before the survey). Fourteen percent of children had a sufficiently varied diet in which they had been given foods from the appropriate number of food groups, and $45 \%$ had been fed the least possible number of times appropriate for their age [17].
Despite a few local studies conducted in different places in the country which explores feeding practice generally [18-20]: there is a shortage of evidence in assessing sick child feeding practice and factors associated with. Hence, this study was aimed to assess sick child feeding practice and associated factors among mothers of under 24-month-old child in Burayu town.

\section{Methods and Materials}

2.1. Study Design and Area. A facility-based cross-sectional study was conducted from April to May 15, 2015. The study was conducted at public health facilities found in Burayu town which is located at $12 \mathrm{~km}$ west to Addis Ababa. The total population of the town was estimated to be 63,873 of whom 31,504 are men and 32369 women [21]. There are 2 health centers and 2 health posts owned by the government, and 40 clinics owned by private organizations. The majority of the town's population receive service from government-owned health facilities.

2.2. Inclusion and Exclusion Criteria. All mothers who visited public health centers in Burayu town during the data collection period were included in the study while those mothers who had a serious illness or seriously ill child were excluded from the study.

\subsection{Sample Size, Study Population and Sampling} Procedure. The sample size was calculated using a single population proportion formula by considering the following assumptions: the proportion of mothers who properly practice sick baby feeding was (45.0\%) from a study by Agumasie et al. in Ethiopia [22]. Confidence interval of 95\%, 5\% margin of error and $10 \%$ none-response. A final sample size of 418 mother-child pair was obtained.

Respondents of the study were taken, from each of the two public health centers in Burayu town depending on their predetermined client flow rate. To enroll respondents into the study: first, the average number of mothers who visited maternal and child health clinic daily at the two health centers were identified by referring client registration book for the last two months prior to data collection. Afterward, participants were included proportionally by considering a possible number of client that can be expected in each health center during the data collection period. Systematic random sampling method was used to select study participants. The average daily client flow rate for the first health center was 16 client and 18 for the second which gives an average monthly client flow rate of 480 and 540 respectively. So the sample was taken from each health center proportional to the expected number of client flow rate so, we took 170 and 192 respondent from the first and second health center respectively.

2.4. Operational Definition and Measurement. Dependent (outcome) variable: sick child feeding practice (good/poor)

Sick child-refers to an infant or young child who had either of the common childhood illness like pneumonia or diarrhea and seeks treatment. 
Sick child-feeding practice - refers to routines of feeding a child at the time of illness. To assess these mothers were asked a question on how frequent they fed their child at the time of illness (the correct answer was more than 2-3 meals per day for those aged 6-8 month, more than 3-4 meals per day for those 9-23 months). And for those who were exclusively breastfed mothers who fed more than the normal frequency (8-12 feeds per day) had good sick child-feeding practice whereas those mothers who gave the usual amount of liquids and those giving somewhat less amount and frequency of liquids than usual or withholding feeding were considered as having poor sick baby-feeding practice [11].

2.5. Data Collection Tool and Procedure. Data were collected from mothers of under 24-month-old child by using structured and interviewer-administered questionnaire. The questionnaire was initially prepared in the English language and then translated to the local language (Afan Oromo) and the responses were translated back to English to check for consistency. Four diploma clinical nurse who can fluently speaks the local language and be available throughout the data collection time undertake the face to face interview and one health officer supervised the overall process on a daily basis.

2.6. Data Quality Control. The collected was checked manually for completeness, cleaned and double entered by using Epidata version 3.1. A pretest was done on 5\% of the sample and the result was used to adjust the content and approach of the questionnaire. Two days training was given both for data collectors and supervisor on the whole process of data collection. The supervisor controls the completeness of the questionnaire and consistency of the data and communicates with the principal investigator in cases of difficulties.

2.7. Data Management and Analysis. The data were analyzed using SPSS version 20. Descriptive statistics like frequency distribution, percentage, and means were used to define respondents in relation to pertinent variables and presented using tables and graphs. Variables which showed association with the dependent variable in the bivariate analysis at alpha $<0.25$ were entered into the multivariable logistic regression model. Adjusted Odds ratios (OR) with corresponding 95\% confidence interval was estimated and $p$-value less than 0.05 were used to identify variables that had a statistically significant association with mothers sick child feeding practice in the final model. Multicollinearity among independent variables was tested by computing variance inflation factor and looking standard error. Hence (VIF $>10)$ and $(\mathrm{SE}>2)$ was suggestive of collinearity. Model fitness was tested by Hosmer-Lemeshow goodness of fit test.

2.8. Ethical Clearance. Ethical approval was obtained from Addis Ababa University College Medicine and allied health Science Ethical Review Board. Respondents were given information regarding the purpose of the study and assured that all information they provide will be confidential. All of the respondents signed on the written informed consent form prior to participation.

\section{Result}

3.1. Sociodemographic Characteristics of the Mother and Child. A total of 362 mother-child pair participated in the study with a response rate of $87 \%$. The mean age of respondents was $25.41( \pm$ SD 3.56$)$ and ranges from $15-30$ years. Nearly half (48.1\%) of all respondents had Oromo ethnic group and fortyfour percent of the mothers were orthodox religion followers. Almost all (97\%) of the respondents were married. Majority of the respondents $(88.4 \%)$ had attended formal education (Table 1).

\subsection{Health Care Service Utilization and Obstetrics Related} Characteristics of the Mothers. Almost all of the mothers had antenatal care follow up however only less than half (47\%) of them had got counseling on infant and young child feeding. About $35 \%$ of mothers used bottle for child feeding. Nearly $94 \%$ of mothers gave birth for an index child at health institution and assisted by a health professional (Table 2).

3.3. Sick Child Feeding Practice of Mothers. The current study showed that nearly fifty-four percent of mothers had a good practice of sick child feeding. Hence those mothers feed their sick child more frequently at a time of illness than when they were healthy (Figure 1).

\subsection{Factors Associated with a Sick Infant and Young Child}

Feeding Practice of Mothers. As shown on the table below, mother's occupation, access to counseling of infant and young child feeding and age of the child were factors that have a statistically significant association with mother's sick child feeding practice. Hence, a mother who had got counseling on sick child feeding were almost three times more likely to feed their child appropriately than those who did not get counseling (AOR: 2.95; 95\% CI; 1.78, 4.91). Likewise, housewife mothers were $55 \%$ times less likely to feed their sick child appropriately than those who worked outside the home (AOR: $0.45 ; 95 \%$ CI; $0.26,0.79$ ). Those mothers who have a child aged less than 6 months were $88 \%$ times less likely to provide more frequent feeds to their sick child than those who have a child aged greater than 6 months (AOR: 0.22; 95\% CI; 0.12,0.40) (Table 3).

\section{Discussion}

The current study showed that more than half (53.6\%) of mothers had an appropriate sick infant and young child feeding practice which means, the mentioned proportion of mother provide their child with breast milk or soft and appetizing complementary diet more frequently at the time of illness than when they were normal. A study by Semahagn et al. in Ethiopia showed that $45.0 \%$ of mothers provide to their child more frequent feeds at the time of illness [22]. Which is slightly lower than the finding in the current study. The difference may be attributed to the variation in the time of the two studies. A nearly consistent finding was reported on a study by Giri et al. in which $51.5 \%$ of the mothers increases the frequency of feeding to their sick child [23]. The finding of a study by Dongre et al. in a tribal district of Maharashtra, India 
TABLE 1: Sociodemographic characteristic of respondents $(n=362)$ visiting $\mathrm{MCH}$ units of health centers in Burayu town, Oromia, Ethiopia, 2015.

\begin{tabular}{|c|c|c|c|}
\hline Variables & Category & Frequency $(n)$ & Percent (\%) \\
\hline \multirow{5}{*}{ Mother's age } & $15-19$ & 3 & 0.80 \\
\hline & $20-24$ & 151 & 41.7 \\
\hline & $25-29$ & 161 & 44.5 \\
\hline & $30-34$ & 42 & 11.6 \\
\hline & $\geq 35$ & 5 & 1.40 \\
\hline \multirow{6}{*}{ Ethnicity } & Oromo & 174 & 48.0 \\
\hline & Amhara & 42 & 11.6 \\
\hline & Tigre & 18 & 5.00 \\
\hline & Gurage & 81 & 22.4 \\
\hline & Wolayita & 34 & 9.40 \\
\hline & Other & 13 & 3.60 \\
\hline \multirow{4}{*}{$\begin{array}{l}\text { Mother's } \\
\text { religion }\end{array}$} & Orthodox & 160 & 44.2 \\
\hline & Muslim & 90 & 29.3 \\
\hline & Protestant & 106 & 59.4 \\
\hline & Other & 6 & 1.70 \\
\hline \multirow{4}{*}{ Marital status } & Married & 351 & 97.0 \\
\hline & Single & 8 & 2.20 \\
\hline & Divorced & 2 & 0.60 \\
\hline & Widowed & 1 & 0.30 \\
\hline \multirow{2}{*}{$\begin{array}{l}\text { Mother's } \\
\text { occupation }\end{array}$} & Housewife & 266 & 73.5 \\
\hline & $\begin{array}{l}\text { Working } \\
\text { outside }\end{array}$ & 96 & 26.5 \\
\hline \multirow{2}{*}{$\begin{array}{l}\text { Mothers } \\
\text { education }\end{array}$} & $\begin{array}{l}\text { Formal educa- } \\
\text { tion }\end{array}$ & 320 & 88.4 \\
\hline & $\begin{array}{l}\text { No formal } \\
\text { education }\end{array}$ & 42 & 11.6 \\
\hline \multirow{3}{*}{$\begin{array}{l}\text { Monthly } \\
\text { income (ETB)* }\end{array}$} & $\leq 1000$ & 147 & 40.6 \\
\hline & $1001-2000$ & 114 & 31.5 \\
\hline & $\geq 2001$ & 101 & 27.9 \\
\hline
\end{tabular}

${ }^{*}$ Ethiopian birr.

that investigated household practices for the sick child also showed, that, the status of some desired household practices such as frequent feeding and giving extra fluid to drink during episodes of illness was poor [24].

The finding of our study revealed that $33.7 \%$ of mothers provide their children with less frequent feeds during illness almost similar finding was reported on study in Tanzania which revealed that, some (35\%) of mothers offered their children less amount of breast milk or nonbreast milk liquids (24\%) because of the child's refusal to feed during illness [25]. Consistent to this finding, a study by Benakappa et al. that evaluates beliefs of caregivers regarding diet during childhood illness; showed that a child must be fed less during illness [26] and $21 \%$ of mothers in a study by Giri et al. believed that breastfeeding should be decreased during illness [23].

In the current study, working mothers were more likely to continue feeding and gave more frequent feeds than housewives consistently, the finding of a study by Dongre et al. which stated that working mothers more commonly continued breastfeeding and gave more frequent feed than their counterpart at the time of illness [24]. This may be attributed to the
TABLE 2: Health care service utilization and obstetrics related characteristics of the mothers of under 24-month-old child $(n=362)$ visiting the $\mathrm{MCH}$ unit of health centers in Burayu town, Oromia, Ethiopia, 2015.

\begin{tabular}{|c|c|c|c|}
\hline Variables & Category & Frequency $(n)$ & Percent (\%) \\
\hline \multirow{3}{*}{ Parity } & $1-2$ & 147 & 40.6 \\
\hline & $3-4$ & 114 & 31.5 \\
\hline & $5 \&$ above & 101 & 27.9 \\
\hline \multirow{2}{*}{ ANC follow up } & Yes & 353 & 97.5 \\
\hline & No & 9 & 2.5 \\
\hline \multirow{2}{*}{$\begin{array}{l}\text { Receive } \\
\text { counseling on } \\
\text { IYCF }\end{array}$} & Yes & 169 & 46.7 \\
\hline & No & 193 & 53.3 \\
\hline \multirow{4}{*}{$\begin{array}{l}\text { Source of } \\
\text { information on } \\
\text { sick baby } \\
\text { feeding }\end{array}$} & $\begin{array}{c}\text { Health } \\
\text { professionals }\end{array}$ & 266 & 62.4 \\
\hline & $\begin{array}{l}\text { Health } \\
\text { extension } \\
\text { workers }\end{array}$ & 24 & 6.6 \\
\hline & Mass media & 43 & 11.9 \\
\hline & Other & 29 & 9.1 \\
\hline \multirow[b]{2}{*}{ Place of delivery } & Home & 20 & 5.5 \\
\hline & $\begin{array}{c}\text { Health } \\
\text { institution }\end{array}$ & 342 & 94.5 \\
\hline \multirow{2}{*}{ Bottle feeding } & Yes & 127 & 35.1 \\
\hline & No & 235 & 64.9 \\
\hline \multirow{3}{*}{ Birth attendant } & TBA & 7 & 1.9 \\
\hline & $\begin{array}{c}\text { Health } \\
\text { professional }\end{array}$ & 343 & 94.7 \\
\hline & Relatives & 12 & 3.3 \\
\hline \multirow{3}{*}{$\begin{array}{l}\text { Accesses to } \\
\text { media }\end{array}$} & Radio & 278 & 76.8 \\
\hline & Television & 248 & 68.5 \\
\hline & $\begin{array}{c}\text { Magazine/ } \\
\text { books/news } \\
\text { paper }\end{array}$ & 54 & 14.9 \\
\hline
\end{tabular}

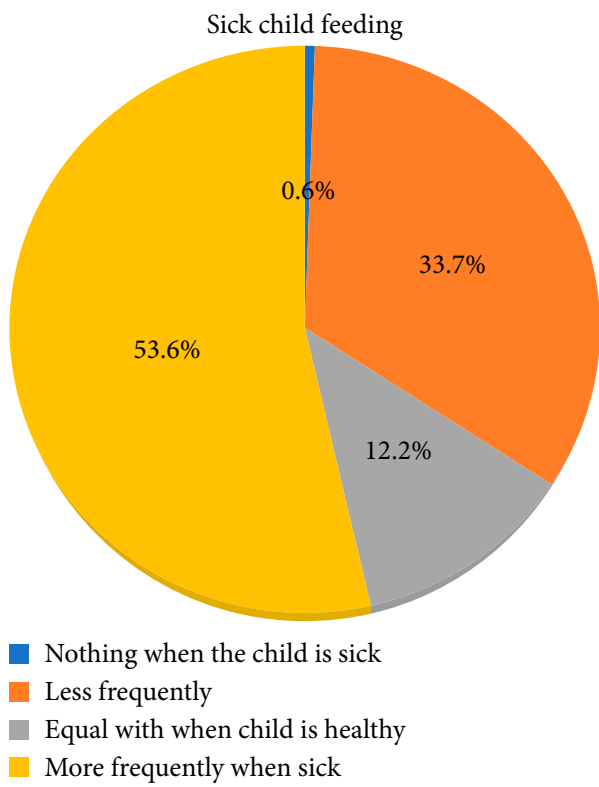

FIGURE 1: Sick child feeding practice of mothers in Burayu town, Oromia, Ethiopia, 2015. 
TABLE 3: Bivariate and multivariable analysis of sick child feeding practice and associated factors among mothers of under 24-month-old child visiting the $\mathrm{MCH}$ unit of health centers in Burayu town, Oromia, Ethiopia, 2015.

\begin{tabular}{|c|c|c|c|c|}
\hline \multirow{2}{*}{ Variables } & \multicolumn{2}{|c|}{ Sick child feeding practice } & \multicolumn{2}{|c|}{$95 \% \mathrm{CI}$} \\
\hline & Good & Poor & COR & AOR \\
\hline \multicolumn{5}{|l|}{ Occupation of mother } \\
\hline Housewives & $158(59.4)$ & $108(40.6)$ & $2.14(1.32,3.43)$ & $0.45(0.26,0.79)^{*}$ \\
\hline Working mothers & $39(40.6)$ & $57(59.4)$ & 1 & 1 \\
\hline \multicolumn{5}{|c|}{ Receive counseling on IYCF } \\
\hline Yes & $113(66.9)$ & $56(33.1)$ & $2.61(4.01,11.70)$ & $2.95(1.78,4.90)^{*}$ \\
\hline No & $84(43.5)$ & $109(56.5)$ & 1 & 1 \\
\hline \multicolumn{5}{|l|}{ Child age } \\
\hline$<6$ months & $42(28.7)$ & $91(71.3)$ & $0.23(0.13,0.39)$ & $0.22(0.12,0.40)^{*}$ \\
\hline 6-12 months & $71(68.9)$ & $32(31.1)$ & 1 & 1 \\
\hline$\geq 12$ & $80(63.5)$ & $46(36.5)$ & 1 & 1 \\
\hline \multicolumn{5}{|l|}{ Monthly income } \\
\hline$<1000$ & $66(44.9)$ & $81(55.1)$ & $0.87(0.68,1.128)$ & \\
\hline $1000-2000$ & $46(40.4)$ & $68(59.6)$ & 1 & \\
\hline$\geq 2000$ & $53(52.5)$ & $48(47.5)$ & 1 & \\
\hline \multicolumn{5}{|l|}{ Mothers education } \\
\hline Formal education & $172(53.8)$ & $148(46.3)$ & $0.79(0.38,1.44)$ & \\
\hline No formal education & $25(61.0)$ & $17(39.0)$ & 1. & \\
\hline
\end{tabular}

${ }^{*} P<0.05$. IYCF- infant and young child feeding.

difference in the level of education and understanding of working mothers as compared to housewives. Even though working mothers spent most of their day out of home, they strongly adhere to the providers counseling and look after their baby at the time of illness and feed them properly than a housewife.

The finding of our study showed that only $0.6 \%$ mothers stopped feeding the baby at the time of illness while a study by Gupta et al. that investigates the first action of mothers for a child with diarrhea reported $7.8 \%$ mothers to stop feeds that, includes breast-feeds and other fluids [27]. The discrepancies may possibly be supported by the difference in time between the two studies, the social and cultural variation among respondents.

In the present study mothers of younger infants were less likely to breastfeed more frequently than mothers of older children who gave more complementary foods during and after an illness. This may be attributed to the poor appetite of infants induced by illness and dependency on only breast milk among infants who didn't start complementary foods. However, the mother's of younger children those who start complementary food can give different types and appetizing complementary food items in addition to breastmilk.

The primary strength of this study is the application of a proper model for analysis and sampling which is representative of the target population. The study also had limitation like the inability to establishing a causal relationship between the explanatory and outcome variable due to the cross-sectional nature of the study. Besides we fail to observe the practice of mothers while they provide feeds exactly at the time of illness despite their response of how frequent they feed their child both when they are healthy and sick. These drawbacks will deter the implication of our finding, so the further study should be carried out to explore the detailed practice by observing the normal meal frequency given to the baby at the time of illness.

\section{Conclusion and Recommendation}

There is increasing dissemination of information on infant and young child feeding (IYCF) behaviors and practices in Ethiopia. However, still, there is limited information about infant and young child feeding at the time of illnesses. Evidence showed that implementation of IYCF behaviors and practices during common childhood illnesses are far from optimal. In general, sick children continue to be breastfed. However, few are breastfed more frequently to compensate for the additional fluid and nutrient requirements which are associated with the illnesses, while a significant proportion of children were breastfed less frequently than usual.

Restriction or withdrawal of breastfeeding or complementary foods during illness is common among respondents, as a result of children's loss of appetite (supposed or actual), and poor awareness of caregivers' about the feeding needs of sick children. Consequently, many sick children were fed less frequently during illness. It would be better if the IYCF behaviors and practices could be strengthened specifically focusing on feeding during and after a common childhood illness.

\section{Abbreviations}

ANC: $\quad$ Antenatal care

PNC: Postnatal care

EDHS: $\quad$ Ethiopian demographic and health survey

WHO: World health organization

AOR: $\quad$ Adjusted odds ratio

CI: $\quad$ Confidence interval

IYCF: Infant and young child feeding

UNICEF: United nations international children's emergency fund. 


\section{Data Availability}

You can have the data set used and/or analyzed during the current study from the following authors (Hiwot Tadesse, Nega Degefa) on a reasonable request.

\section{Ethical Approval}

An institutional review board of Addis Ababa University College of Medicine and Health science had approved this study. An official letter that, explain aim and justification of the study was written by Addis Ababa University College of medicine and health science research coordination office to the town's health office and the letter of support from the town's health office submitted to health facilities of Burayu town. Permission letter was secured from the head of respective health centers. A face to face exit interview was undertaken after taking a verbal informed consent from the mother visiting maternal and child health clinic. To safeguard confidentiality the questionnaires were filled anonymously.

\section{Conflicts of Interest}

All the authors declare that they have no conflicts of interest.

\section{Authors' Contributions}

Hiwot Tadesse, Nega Degefa, and Fekadu Aga had participated in conceiving the study and wrote the proposal. Hiwot Tadesse had also supervised the data collection process frequently during the study. Hiwot Tadesse, Nega Degefa had involved in the analysis and interpretation of the finding. All the authors had read and approved the final draft of this manuscript.

\section{Funding}

All the authors declared that there was no source of financial support to carry out the study.

\section{Acknowledgments}

First and for most, we would like to forward our gratitude to Addis Ababa University College of Medicine and Health Science for offering this opportunity to carry out this research project. Authors are glad to forward their admiration for data collectors, supervisors and different stakeholders who helped during the data collection period.

\section{References}

[1] World Health Organization, Guiding Principles for Feeding Nonbreastfed Children 6-24 Months of Age, 2005.

[2] C. G. Victora, M. de Onis, P. C. Hallal, M. Blössner, and R. Shrimpton, "Worldwide timing of growth faltering: revisiting implications for interventions," Pediatrics, vol. 125, no. 3, pp. e473-e480, 2010.
[3] N. S. Scrimshaw, C. E. Taylor, J. E. Gordon, and World Health Organization, Interactions of Nutrition and Infection, World Health Organization, Geneva, 1968.

[4] World Health Organization, Infant and Young Child Feeding: Model Chapter for Textbooks for Medical Students and Allied Health Professionals, World Health Organization, Geneva, 2009.

[5] World Health Organization and UNICEF, Global Strategy for Infant and Young Child Feeding, World Health Organization, Geneva, 2003.

[6] World Health Organization, Indicators for Assessing Infant and Young Child Feeding Practices: Part 1: Definitions, World Health Organization, Washington, DC, USA, 2008.

[7] K. H. Brown, "Diarrhea and malnutrition," The Journal of Nutrition, vol. 133, no. 1, pp. 328S-332S, 2003.

[8] J. K. Gulati, "Child malnutrition: trends and issues," The Anthropologist, vol. 12, no. 2, pp. 131-140, 2010.

[9] P. Ramachandran and H. S. Gopalan, "Undernutrition \& risk of infections in preschool children," The Indian Journal of Medical Research, vol. 130, no. 5, pp. 579-583, 2009.

[10] C. G. Neumann, M. Marquardt, and N. O. Bwibo, "The impact of morbidity on food intake in rural Kenyan children," South African Journal of Clinical Nutrition, vol. 25, no. 3, pp. 142-148, 2012.

[11] K. Dewey, Guiding Principles for Complementary Feeding of the Breastfed Child, World Health Organization, Washington, DC, USA, 2002.

[12] K. H. Brown, J. M. Peerson, J. Rivera, and L. H. Allen, "Effect of supplemental zinc on the growth and serum zinc concentrations of prepubertal children: a meta-analysis of randomized controlled trials," The American Journal of Clinical Nutrition, vol. 75, no. 6, pp. 1062-1071, 2002.

[13] World Health Organization, Technical Updates of the Guidelines on the Integrated Management of Childhood Illness (IMCI): Evidence and Recommendations for Further Adaptations, World Health Organization, Geneva, 2005.

[14] World Health Organization, Essential Nutrition Actions: Improving Maternal, Newborn, Infant and Young Child Health and Nutrition, World Health Organization, Geneva, 2013.

[15] World Health Organization, Tracking Progress for Breastfeeding Policies and Programmes: Global Breastfeeding Scorecard 2017, World Health Organization, Geneva, 2017.

[16] J. Lawn and K. Kerber, Opportunities for Africa's newborns. Cape Town: The Partnership for Maternal, Newborn \& Child Health, World Health Organization, Geneva, 2006.

[17] Central Statistical Agency and ICF, Demographic and Health Survey 2016, CSA and ICF, Addis Ababa, Ethiopia, 2017.

[18] T. Yeneabat, "Determinants of cessation of exclusive breastfeeding in Ankesha Guagusa Woreda, Awi Zone, Northwest Ethiopia: a cross-sectional study," Tropical Medicine \& International Health, vol. 20, p. 364, 2015.

[19] D. Tamiru, T. Belachew, E. Loha, and S. Mohammed, "Suboptimal breastfeeding of infants during the first six months and associated factors in rural communities of Jimma Arjo Woreda, Southwest Ethiopia," BMC Public Health, vol. 12, no. 1, p. 363, 2012.

[20] A. Lenja, T. Demissie, B. Yohannes, and M. Yohannis, "Determinants of exclusive breastfeeding practice to infants aged less than six months in Offa district, Southern Ethiopia: a cross-sectional study," International Breastfeed Journal, vol. 11, p. 32, 2016. 
[21] Population Census Commission, Summary and Statistical Report of the 2007 Population and Housing Census. Population Size by Age and Sex, FAO, Rome, 2008.

[22] A. Semahegn, G. Tesfaye, and A. Bogale, "Complementary feeding practice of mothers and associated factors in Hiwot Fana Specialized Hospital, Eastern Ethiopia," The Pan African Medical Journal, vol. 18, , 2014.

[23] P. A. Giri and D. B. Phalke, "Beliefs and practices regarding diet in common childhood illnesses among rural caregivers," Journal of Medical Nutrition and Nutraceuticals, vol. 3, no. 2, pp. 99-101, 2014.

[24] A. Dongre, P. Deshmukh, and B. Garg, "Childhood morbidity, household practices and health care seeking for sick children in a tribal district of Maharashtra, India," Indian Journal of Medical Sciences, vol. 64, no. 1, p. 7, 2010.

[25] J. L. Kinabo, A. W. Mwanri, P. S. Mamiro et al., "Infant and young child feeding practices on Unguja Island in Zanzibar, Tanzania: a ProPAN based analysis," Tanzania Journal of Health Research, vol. 19, no. 3, , 2017.

[26] A. D. Benakappa and P. Shivamurthy, "Beliefs regarding diet during childhood illness," Indian Journal of Community Medicine: Official Publication of Indian Association of Preventive \& Social Medicine, vol. 37, no. 1, p. 20, 2012.

[27] R. K. Gupta and R. Gupta, "First action management of acute diarrhea in children by rural mothers," JK Science, Journal of Medical Education and Research, vol. 2, no. 2, pp. 96-98, 2000. 


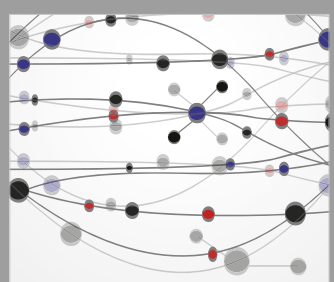

The Scientific World Journal
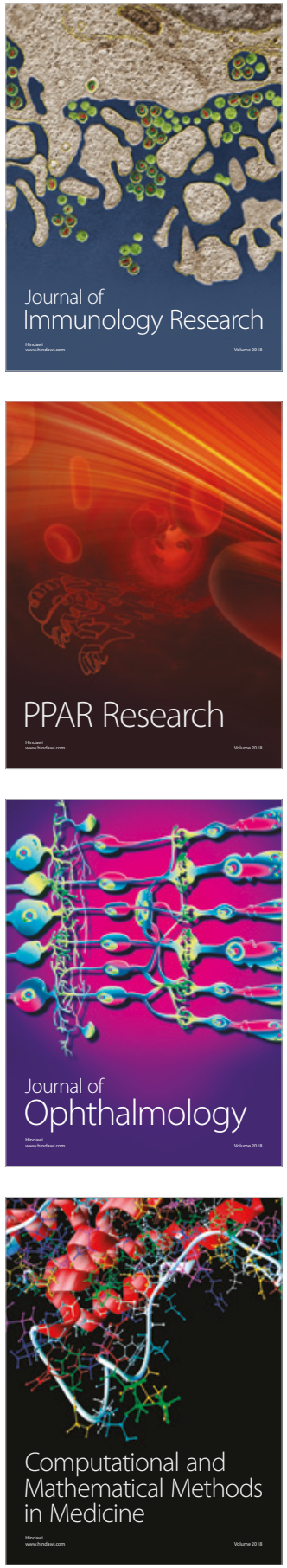

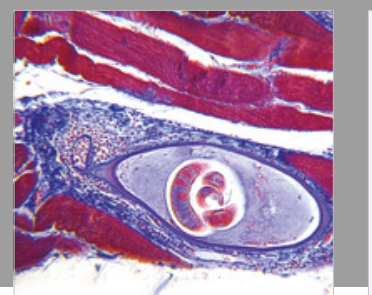

Gastroenterology Research and Practice

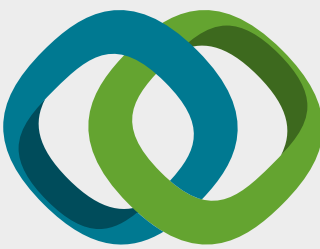

\section{Hindawi}

Submit your manuscripts at

www.hindawi.com
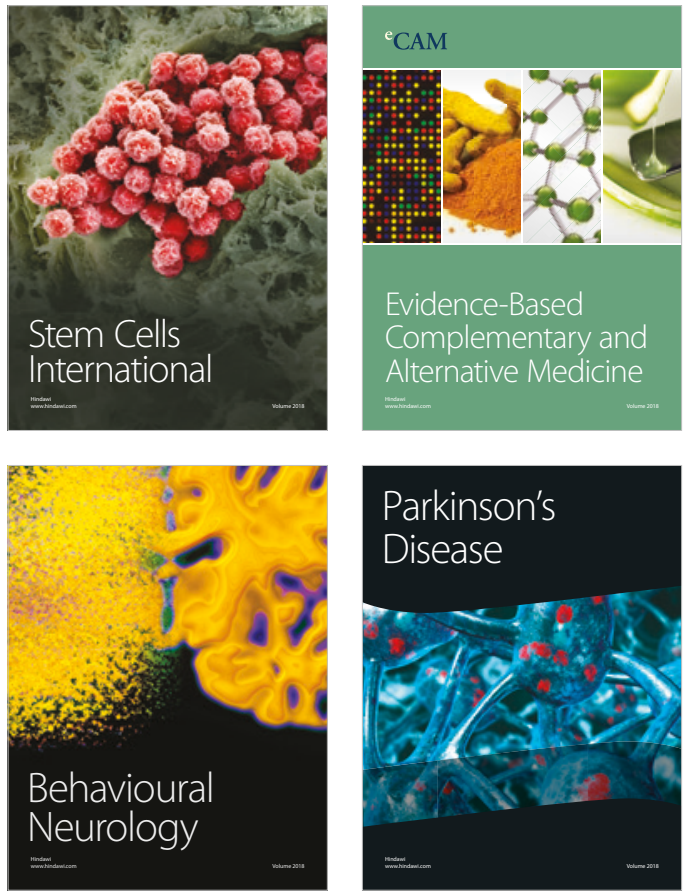

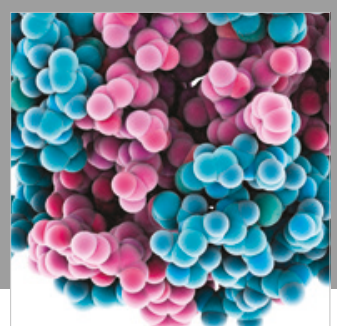

ournal of

Diabetes Research

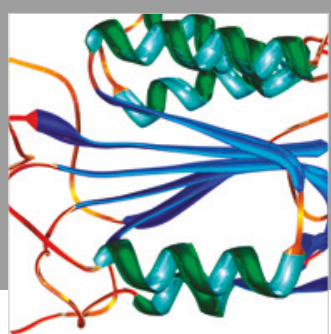

Disease Markers
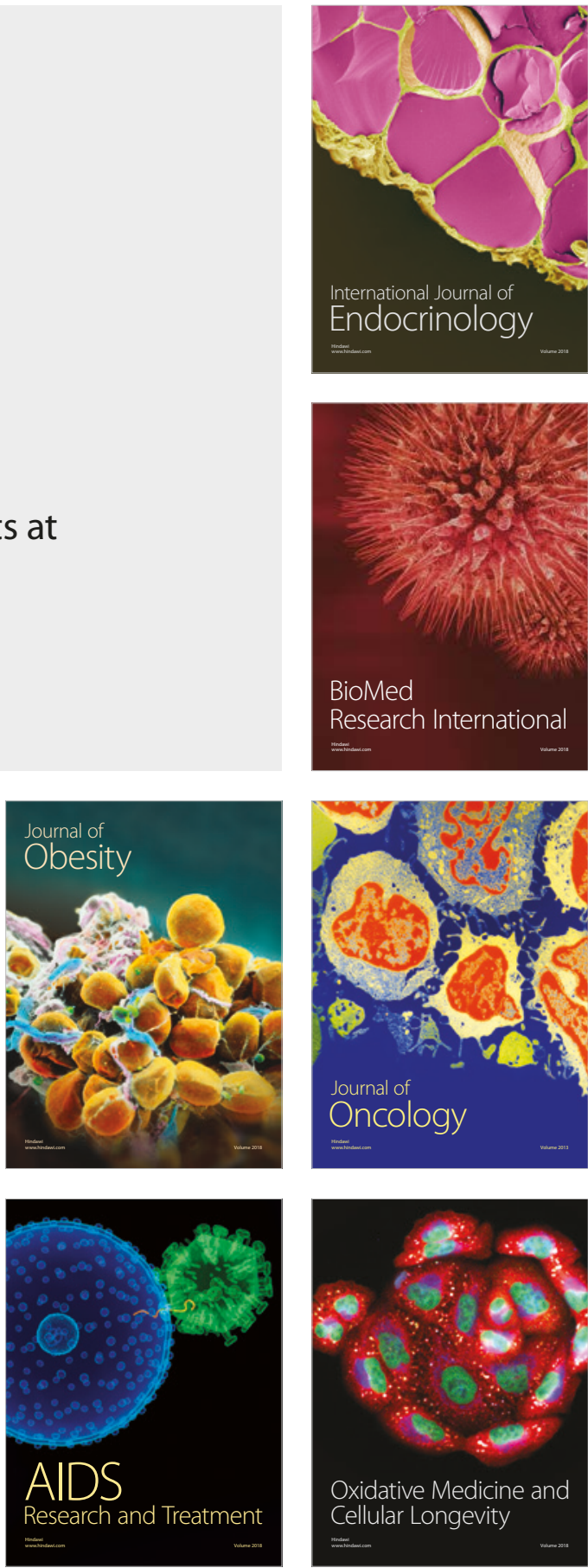